\title{
Nanomedicine for immunotherapy
}

\author{
"While most of the work with nanomedicines tries to \\ target the tumor directly, there are definitely much \\ more opportunities for nanoparticular carrier systems \\ to improve therapeutic outcome of conventional \\ interventions."
}

First draft submitted: 4 August 2016; Accepted for publication: 19 August 2016; Published online: 26 September 2016

Keywords: elimination of tolerance $\bullet$ activation $\bullet$ immune system $\bullet$ nanoparticles

The starting point for this special volume was discussions parallel to the meeting with the title: "Quo Vadis Nanomedicine?" organized in 2014 in Exeter, UK by M Barz, $\mathrm{R}$ Luxenhofer and M Schillmeier, which led to a special volume with the same title in this journal [1].

While most of the work with nanomedicines tries to target the tumor directly, there are definitely much more opportunities for nanoparticular carrier systems to improve therapeutic outcome of conventional interventions. One example is immune therapies, which can be used to fight either chronic infections, allergies or even cancer. Especially in cancer therapy the use of immune therapy seems highly promising following the old idea of a vaccination against tumor associated antigens. The immune system, however, is equipped with different levels of self-control to avoid autoimmune responses. Tumors, for example, exploit these tolerance mechanism (or at least any successfully expanding tumor has found a way to induce tolerance). Thus, it is not sufficient to only activate the immune system against a tumor associated antigen, but the elimination of immune tolerance is of equal importance (see the Editorial by Grabbe et al. [2] and the contribution by Jonuleit et al. [3]).

In this respect, we combined in this special volume Research Papers, Perspectives and Reviews, which address different aspects of cancer immune therapies, from the elimination of immune tolerance to antigen-selective activation. These contributions are completed by a report about concepts for immuneimaging and a case study about RNALipoplexes for the treatment of melanoma.

At first Mailänder and Tonigold underline differences in cellular uptake mechanisms for nanoparticles in dendritic cells, which determine the intracellular fate of nanoparticle and cargo and are thus highly relevant for the effective delivery of nanosized therapeutics [4].

Next Foerster et al. evaluate a new carrier system with minimal toxicity, which is based on cross-linked dextran for hepatic drug delivery and the addressing of liver macrophages [5].

De Geest and coworkers present a new synthetic route to core cross-linked polymer micelles, which can be used as welldefined vaccines co-delivering antigen and adjuvant [6].

Bros, Zentel and coworkers describe the synthesis of micelles from block copolymers with pHPMA as the hydrophilic corona and their functionalization with mannose [7]. These micelles bound DC only when conjugated with mannose, and in a mannose receptor-specific manner leading to internalization. This allows a selective DC activation by loading of the micelles with hydrophobic L18-MDP.
Rudolf Zentel

Institute of Organic Chemistry, Johannes Gutenberg-University Mainz, Duesbergweg 10-14, D-55099 Mainz, Germany

zentel@uni-mainz.de 
Shen et al. study dextran-coated colloidal superparamagnetic nanoparticles, which are triple functionalized with immunostimulatory $\mathrm{CpG}-\mathrm{ODN}$, antiDEC205 antibody (targeting to DCs) and ovalbumin as a model antigen to induce antigen-specific $\mathrm{T}$-cell responses and antitumor responses in in vitro and in vivo experiments [8]. These particles induce a strong activation of the immune system in vitro and lead to a tumor growth arrest in vivo.

“

"...the use of immune therapy seems highly promising following the old idea of a vacçination against tumor associated antigens.

Tüttenberg, Steinbrink and Schuppan review the role of myeloid cells as orchestrators of the tumor microenvironment. Due to their role in immunosuppression they are new targets for nanoparticular cancer therapy [9].

Jonuleit, Bopp and Becker review the role of regulatory $\mathrm{T}$ cells - another class of cells controlling

\section{References}

1 Barz M, Luxenhöfer R, Schillmeier M. Quo vadis nanomedicine? Nanomedicine 10(20), 3089-3091 (2015).

2 Grabbe S, Landfester K, Schuppan D, Barz M, Zentel R. Nanoparticles and the immune system: challenges and opportunities. Nanomedicine 11(20), 2621-2624 (2016).

3 Jonuleit H, Bopp T, Becker C. Regulatory T cells as potential cellular targets for functionalized nanoparticles in cancer therapy. Nanomedicine 11(20), 2699-2709 (2016).

4 Mailänder V, Tonigold M. Endocytosis and intracellular processing of nanoparticles in dendritic cells: routes to effective immuno-nanomedicines. Nanomedicine 11(20), 2625-2630 (2016).

5 Foerster F, Bamberger D, Schupp J et al. Dextran-based therapeutic nanoparticles for hepatic drug delivery. Nanomedicine 11(20), 2663-2677 (2016).

6 Vanparijs N, Nuhn L, Paluck SJ et al. Core/shell proteinreactive nanogels via a combination of RAFT polymerization and vinyl sulfone post-modification. Nanomedicine 11(20), 2631-2645 (2016) immune suppression - as potential cellular targets for functionalized nanoparticles in cancer therapy [3].

Diken, Miederer et al. present concepts for immunoimaging as a noninvasive in vivo preclinical method for imaging of the status of the immune system [10].

Finally, Grabbe, Langguth, Sahin and coworkers report a case study of RNA-Lipoplexes for the treatment of melanoma as personalized nano-sized cancer vaccines [11].

\section{Financial \& competing interests disclosure}

The author has no relevant affiliations or financial involvement with any organization or entity with a financial interest in or financial conflict with the subject matter or materials discussed in the manuscript. This includes employment, consultancies, honoraria, stock ownership or options, expert testimony, grants or patents received or pending, or royalties.

No writing assistance was utilized in the production of this manuscript.

7 Mohr N, Kappel C, Kramer S, Bros M, Grabbe S, Zentel $\mathrm{R}$. Targeting cells of the immune system: mannosylated HPMA-LMA block-copolymer micelles for targeting of dendritic cells. Nanomedicine 11(20), 2679-2697 (2016).

8 Shen L, Krauthäuser S, Fischer K et al. Vaccination with trifunctional nanoparticles that address $\mathrm{CD} 8^{+}$dendritic cells inhibits growth of established melanoma. Nanomedicine 11(20), 2647-2662 (2016).

9 Tuettenberg A, Steinbrink K, Schuppan D. Myeloid cells as orchestrators of the tumor microenvironment: novel targets for nanoparticular cancer therapy. Nanomedicine 11(20), 2735-2751 (2016).

10 Diken M, Pektor S, Miederer M. Harnessing the potential of non-invasive in vivo preclinical imaging of the immune system: challenges and prospects. Nanomedicine 11(20), 2711-2722 (2016)

11 Langguth P, Grabbe S, Haas H, Sahin U. Nanoparticulate personalized cancer vaccines in clinical applications: RNAlipoplexes for the treatment of melanoma. Nanomedicine 11(20), 2723-2734 (2016). 